\title{
Robust feedback control of continuously operated convective dryers for particulate materials
}

Seidel, C. ; $^{\text {; Neugebauer, C. }}{ }^{\text {; }}$ Dürr, R. ; Bück, A $^{\mathrm{c}^{*}}$

a Automation/Modelling, Otto von Guericke University Magdeburg, Magdeburg, Germany

${ }^{\mathrm{b}}$ KU Leuven, Leuven, Belgium

${ }^{\mathrm{c}}$ Institute of Particle Technology, FAU Erlangen-Nuremberg, Erlangen, Germany

*E-mail of the corresponding author: andreas.bueck@fau.de

\begin{abstract}
Convective drying is a common process for the treatment of particulate solids also operated in continuous mode. Continuous operation is characterised by operation in steady-state with constant product throughput and constant product quality. Due to external influences, i.e. seasonal or local variations in the properties of the initial wet material, deviations in the product quality can result, for example over- or under-drying, or on-set of unwanted reaction. In this contribution a new feedback control concept is developed that is robust with respect to variations and uncertainties in the drying kinetics of the material and can reject and attenuate process disturbances.
\end{abstract}

Keywords: Process control; drying kinetics; robustness; fluidized bed; yeast. 


\section{Introduction}

Drying, the removal of liquid from a solid material, is one of the major unit operations in solids processing, e.g. chemicals, pharmaceuticals, food and $\operatorname{paper}^{[1,2]}$. The liquid can be removed mechanically, e.g. draining, wringing, filtering, or thermally by induction of a phase change, e.g. by evaporation or sublimation of the liquid. Thermal drying is extensively used and is one of the most energy-intensive processes, taking up approximately $10-25 \%$ of a nation's energy output ${ }^{[1]}$.

Considering convective drying of particulate solids, characteristic evaporation rates as a function of moisture content $\mathrm{X}$, i.e. the mass of liquid per mass of dry solid, are shown in Fig. 1: For moisture contents $\mathrm{X}>\mathrm{X}_{\mathrm{cr}}$, where $\mathrm{X}_{\mathrm{cr}}$ is the material-specific critical moisture content, a constant evaporation rate is observed; for moisture contents $\mathrm{X}_{\mathrm{hyg}}<\mathrm{X}<\mathrm{X}_{\mathrm{cr}}$ a material-dependent falling rate is observed. If the moisture content reaches $\mathrm{X}_{\mathrm{hyg}}$, thermodynamic (adsorption) equilibrium is attained and the evaporation rate vanishes.

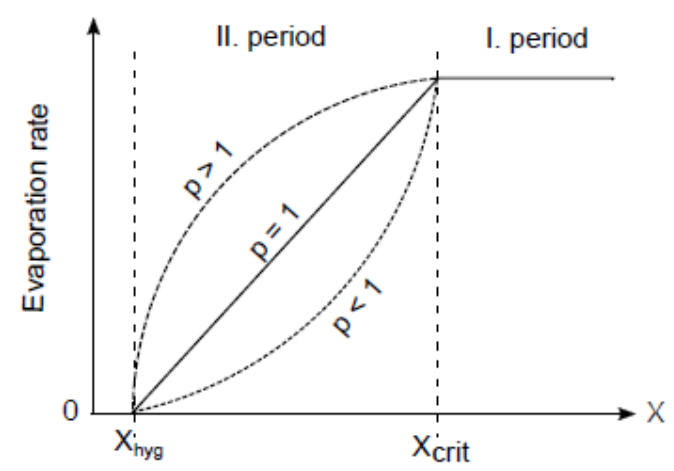

Fig. 1. Schematic overview of experimentally observed evaporation rates in drying of solids.

The first (constant) drying period refers to the surface-wet particle, i.e. direct heat and mass transfer between liquid and gas. In this period, the heat and mass transfer is gas-side controlled, i.e. the gas conditions, e.g. temperature and mass flow rate, directly determine the evaporation rate. In the second drying period, the falling rate period, moisture is mostly located in the (porous) interior -- heat and gas now have to penetrate the solid by conduction and diffusion first in order to evaporate the liquid; also the vapour has to be transported to the particle surface, e.g. by vapour diffusion or capillary pumping, before it can be taken up by the main gas flow. The farther inside the moisture is located, the longer diffusion and conduction processes take and thereby reduce the evaporation rate in the second drying period. 
From this phenomenological description, two different aspects can be identified that influence the drying process: (1) gas-side conditions and (2) material properties. A variation of gas-side conditions only will yield different evaporation rates; the material-specific part, however, remains the same, i.e. the evaporation rates are qualitatively but not quantitatively similar.

An approachto model this effect is given by the concept of normalized drying curve $v$ (NDC), originally developed by van $\mathrm{Meel}^{[3]}$. Here, the second drying period rate is expressed in terms of the (known and constant) gas-side controlled first drying period rate. Main advantage of the NDC is that it can be obtained directly from considerably simpler measurements than effective diffusivity; main drawback is that it only allows describing the evolution of the average moisture content, X. However, as this value is of primary concern in many practical applications, the concept of normalised drying curve has found widespread use for dryer design, optimisation and troubleshooting.

The normalised drying curve is defined as:

$$
\dot{\nu}(\eta)=\frac{\dot{m}_{\text {evap }, I I}}{\dot{m}_{\text {evap }, I}}, \quad \eta=\frac{X-X_{\text {hyg }}}{X_{c r}-X_{\text {hyg }}},
$$

where $\eta$ denotes the normalised moisture content, with values $0 \leq \eta \leq 1$ denoting the second drying period. Given experimental data, the normalised drying curve can be fitted and used for process modelling and feedback controller design as will be shown in the following.

In this work, we present a general approach to robust feedback control of continuously operated convective dryers. In the following section, we present the dynamic drying model equations. Afterwards, the robust controller is designed and its main features are discussed. In the Results section, the performance of the controller, designed for a nominal operating point, is presented with respect to model uncertainties and different operating points. The work closes with Conclusions and Outlook on future work.

\section{Process Modelling}

For the purpose of this work, we pose the following assumptions: (i) The particulate phase in the apparatus can be (at least theoretically) considered as well-mixed. (ii) The gas-phase is also considered as well-mixed, i.e. no spatial gradients. (iii) The particulate phase is either mono-disperse or represented by a constant Sauter mean diameter $\mathrm{d}_{32}$, i.e. a particle size distribution is not considered. Each particle dries as if it were a single particle. (iv) Drying is kinetically-controlled, i.e. drying gas is not close to saturation. (v) Drying takes place under approximately adiabatic conditions, i.e. sufficient insulation of apparatus provided to avoid significant heat loss to the environment. (vi) Particles enter the apparatus 


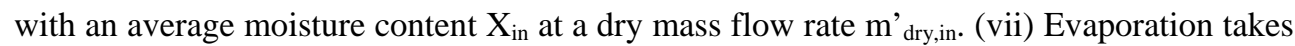
place from the total surface area of all particles. (viii) The average residence time of particles in the continuously operated dryer is $\tau$, which can be regulated, e.g. by speed of conveyor, or hold-up mass control.

Then, starting from a mass balance of the wet solid $\mathrm{m}_{\text {wet }}=\mathrm{X} \mathrm{m}_{\mathrm{dry}}$, the following mass balances for the dry solid (hold-up) and the average moisture content can be derived:

$$
\begin{aligned}
\frac{\mathrm{d} X}{\mathrm{~d} t} & =\left(\dot{m}_{d r y, i n} X_{i n}-\frac{m_{d r y}}{\tau} X-\dot{m}_{\text {evap }}-X \frac{\mathrm{d} m_{d r y}}{\mathrm{~d} t}\right) / m_{d r y}, \\
\frac{\mathrm{d} m_{d r y}}{\mathrm{~d} t} & =\dot{m}_{d r y, i n}-\frac{m_{d r y}}{\tau} .
\end{aligned}
$$

The evaporation rate is written as

$$
\dot{m}_{\text {evap }}=\dot{m}_{\text {evap }, I} A \dot{\nu}=\varrho_{g} \beta\left(Y_{\text {sat }}\left(T_{\text {in }}\right)-Y_{\text {in }}\right) A \dot{\nu}
$$

wherein A denotes the total particle surface area available for evaporation; and $Y_{\text {sat }}-Y_{\text {in }}$ the maximum drying potential of the gas, which is determined by the inlet gas moisture content $Y_{\text {in }}$ and the inlet gas temperature $T_{i n}$. If significant heat losses occur (violation of assumption (v)), then $Y_{\text {sat }}-Y_{\text {in }}$ decreases in value to $Y_{\text {out }}-Y_{\text {in }}$; the model structure, however, does not change.

The total surface area of particles can be determined from the hold-up mass $A=6 m_{\text {dry }} /\left(\rho d_{32}\right)$. The mass transfer coefficient $\beta$ is in general a function of the Sherwood, Reynolds, Prandtl and Schmidt number.

The normalised drying curve is denoted by $v(\eta)$; in order to use the normalised drying curve in process models, some functional form needs to be fitted. In this work, the following form is used:

$$
\dot{\nu}(\eta)=\frac{p \eta}{1+(p-1) \eta}
$$

The virtue of this functional form is that one fitting parameter $\mathrm{p}$ suffices to describe the different curvatures. At $p=1$, corresponding to the linear relation $v(\eta)=\eta$, a change in curvature occurs that can be smoothly related to values $0<\mathrm{p}<1$ and $\mathrm{p}>1$. It has to be noted that all material characteristics are lumped into the parameter $\mathrm{p}$.

Under the stated restrictions, the presented model is able to calculate the dynamic and steady-state behaviour of a continuous convective dryer. 


\section{Robust Controller Design}

The aim of the robust controller is to provide comparable performance of the uncertain drying process with respect to the nominal process conditions and parameters. Specifically, it should provide at different steady-states the required steady-state output (controlled output), provide not too slow process dynamics in doing so, avoid excessive control action, respecting known limits of the actuators and operating conditions, e.g. a maximum operating temperature.

These requirements can be met in (linear) controller design within the $\mathrm{H}_{\infty}$ framework ${ }^{[4]}$. Within this framework, a (linear) controller with transfer function $\mathrm{H}_{\infty}(\mathrm{s})$ is determined such that a) the closed-loop process is asymptotically stable and b) the following functional is minimised:

$$
\min _{H_{\infty} \text { stabilizing }}\left\|\begin{array}{l}
S(s) \cdot W_{1}(s) \\
H_{\infty}(s) S(s) \cdot W_{2}(s) \\
T(s) \cdot W_{3}(s)
\end{array}\right\|_{\infty}
$$

Therein, $\mathrm{s}$ denotes the variable of the Laplace domain, $\mathrm{S}$ denotes the closed-loop sensitivity function, $T^{*}$ the closed-loop complementary sensitivity function, and $W_{i}(i=1,2,3)$ are weight functions.

To assess and quantify the process uncertainty, different models can be used. In this work, we restrict ourselves to the multiplicative uncertainty model: Given a linear open-loop stable nominal transfer function $G_{\text {nom }}(s)$, and a second, uncertain but open-loop stable transfer function $\mathrm{G}(\mathrm{s}) \$$, the multiplicative model uncertainty can be expressed as

$$
\Delta_{m}(s)=\left(G(s)-G_{\text {nom }}(s)\right) G_{\text {nom }}^{-1}(s)
$$

A variation in parameters generates a family of transfer functions $G(s)$ and a set of multiplicative uncertainty models $\Delta_{\mathrm{m}}$ (s). Let the upper-bound of $\Delta_{\mathrm{m}}(\mathrm{s})$ for a given set of variations be denoted by $\Lambda_{\mathrm{m}}(\mathrm{s})$.

For design of performance, the weights $\mathrm{W}_{\mathrm{i}}$ can be used: $\mathrm{W}_{1}$ is used to design good disturbance rejection over a wide frequency region; $\mathrm{W}_{2}$ directly influences the controller gain, thus limiting the control energy; and $\mathrm{W}_{3}$ is used to achieve good reference tracking. For a multiplicative uncertainty model, $\mathrm{W}_{3}(\mathrm{~s})=\Lambda(\mathrm{s})$ is set.

Given the nominal model, the upper bound on model uncertainty and the weight functions, a robustly stabilising feedback controller can be readily computed by established control software, e.g. Matlab’s Control System Toolbox. 


\section{Results}

In the following, specific results of the design and performance of the controller are presented for fluidized bed drying of spherical baker's yeast pellets. Uncertain parameters are: $p$ ranges in the interval $[0.5,2.5]$ with a nominal value of 1.5 , and $X_{\text {crit }}$ the point of onset of the second drying period which is known to slightly depend on drying temperature: $\mathrm{X}_{\text {crit }}$ in the interval $[0.8,1.2]$ with a nominal value of 0.98 .

The manipulated variable is the gas inlet temperature $T_{\text {gas,in, }}$, the controlled output is the average moisture content of the yeast pellets, $\mathrm{X}$, i.e. a SISO control problem. Unmeasured disturbances are the solids and gas inlet moisture contents, $X_{\text {in }}$ and $Y_{\text {in }}$, respectively. For reasons of comparison a standard SISO PI controller and a linear quadratic regulator (LQI) are also designed for the nominal case.

Both controllers are applied to the nonlinear model to assess their performance and robustness of the closed-loop. We start the presentation with the discussion of disturbance rejection. At the nominal steady state, a stepwise disturbance $\left(X_{\text {in }}-15 \%\right)$ is applied at $t=30$ minutes and ends after additional 10 minutes. At 50 minutes a second disturbance $\left(\mathrm{Y}_{\text {in }}+\right.$ $115 \%$ ) is applied for 10 minutes. The closed-loop response under both controllers is shown in Fig. 2. It can be seen that in the nominal scenario, both controllers have comparable performance, i.e. the settling times after a disturbance.

To investigate the impact of the (uncertain) parameter $\mathrm{p}$, additional simulations were performed under otherwise nominal conditions. Figure 3 shows the results of a simulation with the maximum value $\mathrm{p}=2.5$. One can clearly observe that the performance of the $\mathrm{H}_{\infty}$ controller is constant in the parameter range, even at at a different set-point. Contrary, the PI-controller and LQI-controller lose performance at a non-nominal set point and the decrease in performance is higher with increasing value of $\mathrm{p}$.

\section{Conclusions}

We presented an application of robust controller design to a wide range of continuously operated convective drying processes, where the main process uncertainty is in the parameters of the kinetics of the second drying period. We could show that these processes are asymptotically open-loop stable for practically relevant operating parameters. Suitable feedback controllers can be designed in the $\mathrm{H}_{\infty}$ framework given the knowledge of a nominal operating point and bounds on the parameters.

Future work will focus on the extension of this approach to MIMO problems, considering additional manipulated variables, e.g. the gas mass flow rate, and controlled outputs, e.g. product temperature or water activity. 


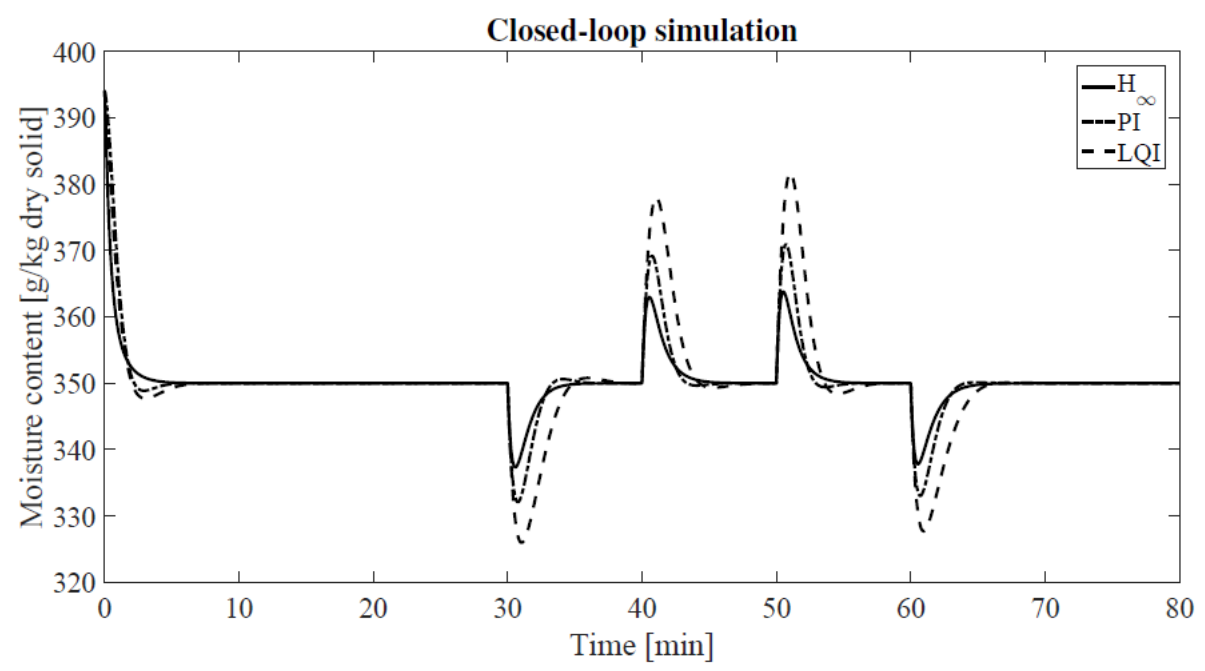

Fig. 2. Response of the controlled nominal system $\left(\mathrm{H}_{\infty}\right.$, PI and LQI controller) for the described disturbance scenario for the set points $X=350$ (g water) $/(\mathrm{kg}$ dry solid).

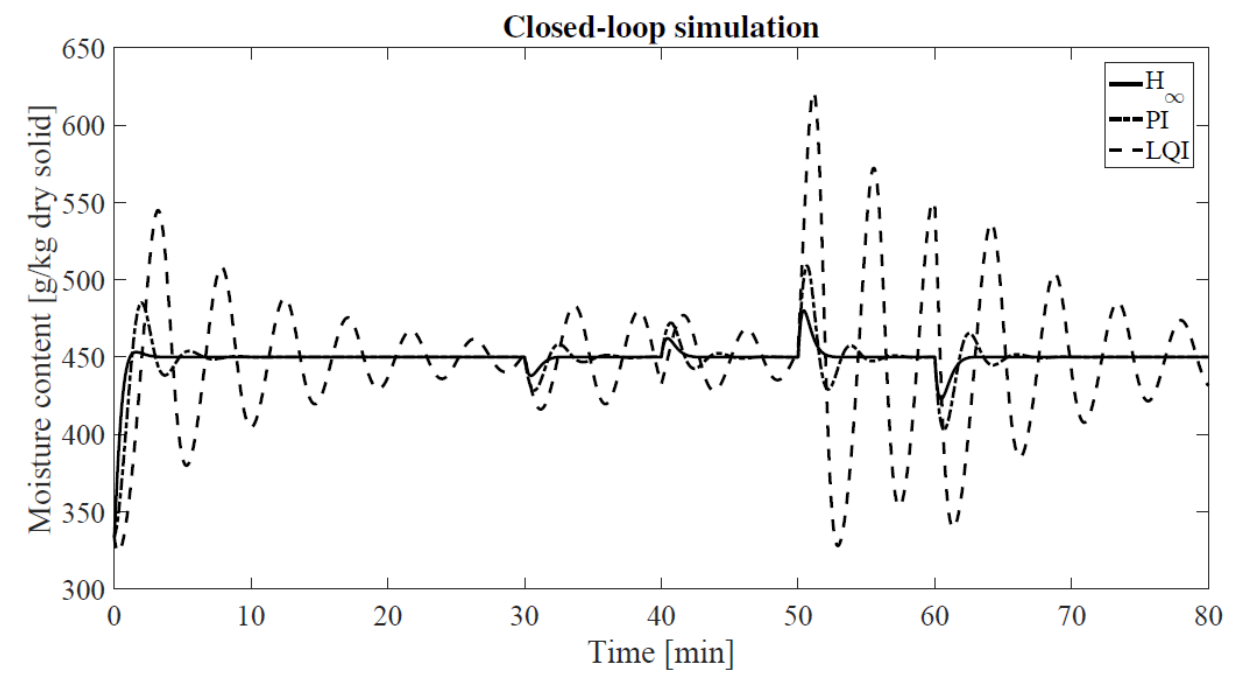

Fig. 3. Response of the controlled system ( $H_{\infty}, P I$ and LQI controller) for the described disturbance scenario for the non-nominal set-point $X=450$ (g water) $/(\mathrm{kg}$ dry solid) and $p=2.5$ (right). 


\section{Main Nomenclature}

$\begin{array}{lll}\text { A } & \text { surface area } & \mathrm{m}^{2} \\ \mathrm{~d} & \text { particle diameter } & \mathrm{m} \\ \mathrm{G}, & \mathrm{S}, & \text { transfer functions } \\ \mathrm{T}^{*} & & \mathrm{~kg} \\ \mathrm{~m} & \text { mass } & \\ \mathrm{p} & \text { parameter of NDC } & \mathrm{s} \\ \mathrm{t} & \text { time } & { }^{\circ} \mathrm{C} \\ \mathrm{T} & \text { temperature } & \\ \mathrm{X} & \text { solid moisture content (d.b.) } & \\ \mathrm{Y} & \text { gas moisture content (d.b.) } & \end{array}$

Greek letters

$\begin{array}{lll}\beta & \text { mass transfer coefficient } & \mathrm{ms}^{-1} \\ \eta & \text { normalised moisture content } & \\ v & \text { normalised drying rate } & \\ \rho & \text { mass density } & \mathrm{kgm}^{-3} \\ \tau & \text { mean residence time } & \mathrm{s}\end{array}$

Subscripts

$\begin{array}{ll}\text { crit } & \text { critical } \\ \text { in } & \text { inlet } \\ \text { hyg } & \text { hygroscopic } \\ \text { sat } & \text { saturation }\end{array}$

\section{References}

[1] Kemp, I. Reducing dryer energy use by process integration and pinch analysis, Drying Technology 2005, 23 (5), 2089-2104.

[2] Tsotsas E. Influence of drying kinetics on partile formation: A personal perspective, Drying Technology 2012, 30 (11), 1167-1175.

[3] van Meel, D. Adiabatic convection batch drying with recirculation of air, Chemical Engineering Science 1958, 9, 36-44.

[4] Zhou, K.; Doyle, J. Essentials of robust control; Prentice Hall: Upper Saddle River, NJ, 1998. 